\title{
The reactive transport of trichloroethene is influenced by residence time and microbial numbers
}

\author{
Haest P.J. ${ }^{1,2}$, J. Philips ${ }^{1}$, D. Springael ${ }^{1}$ and E. Smolders ${ }^{1}{ }^{*}$
}

${ }^{1}$ Division of Soil and Water Management, KU Leuven (University of Leuven), Kasteelpark Arenberg 20, B-3001 Heverlee BELGIUM, Tel: +3216321609 Fax: +3216321997

${ }^{2}$ Current addresses: Flemish Institute for Technological Research (VITO), Boeretang 200, B-2400 Mol, Belgium

Department of Bioscience Engineering, University of Antwerp, Groenenborgerlaan 171, B-2020 Antwerpen, Belgium

*Corresponding author: erik.smolders@ees.kuleuven.be

\begin{abstract}
The dechlorination rate in a flow-through porous matrix can be described by the species specific dechlorination rate observed in a liquid batch unless mass transport limitations prevail. This hypothesis was examined by comparing dechlorination rates in liquid batch with that in column experiments at various flow rates $\left(3-9-12 \mathrm{~cm} \mathrm{~d}^{-1}\right)$. Columns were loaded with an inoculated sand and eluted with a medium containing $1 \mathrm{mM}$ trichloroethene (TCE) for 247 days. Dechlorination in the column treatments increased with decreasing flow rate, illustrating the effect of the longer residence time. Zero ${ }^{\text {th }}$ order TCE or cis-DCE degradation rates were 4-7 fold larger in columns than in corresponding batch systems which could be explained by the higher measured Geobacter and Dehalococcoides numbers per unit pore volume in the columns. The microbial numbers also explained the variability in dechlorination rate among flow rate treatments marked by a large elution of the dechlorinating species' yield as flow increased. Stop flow events did not reveal mass transport limitations for dechlorination. We conclude that flow rate effects on reactive transport of TCE in this coarse sand are explained by residence time and by microbial transport and that mass transport limitations in this porous matrix are limited.
\end{abstract}

\section{KEYWORDS}

Anaerobic column experiment, Dehalococcoides, Geobacter, microbial transport, reactive transport, reductive dechlorination, TCE.

Please cite this article as: P.J. Haest, J. Philips, D. Springael, E. Smolders (2011). The reactive transport of trichloroethene is influenced by residence time and microbial numbers. Journal of Contaminant Hydrology, 119: 89-98. 


\section{Introduction}

Bioremediation of groundwater contaminated with Chlorinated Aliphatic Hydrocarbons (CAHs) is a valuable remediation strategy in addition to physical or chemical remediation technologies (Christ et al. 2005; Ellis et al. 2000; Major et al. 2002; Sleep et al. 2006). The CAHs can be biologically degraded by reductive dechlorination to the harmless ethene (Bradley 2003). The success of bioremediation in an aquifer can be estimated by modelling after obtaining information on degradation rates and the local hydrogeology. The degradation rates can be estimated with batch microcosms. However, degradation rates observed in batch microcosm mostly differ from those observed in situ, likely due to differences in chemical, microbiological or physical factors (Adamson et al. 2003; Yang and McCarty 2002; Carr and Hughes 1998).

Theory on reactive transport states that the biodegradation in a porous matrix with flowthrough is determined by the intrinsic reaction kinetics, the residence time and the number of residing degrading bacteria if those bacteria have equal reactivity to the soluble degradable substrate as in liquid batch microcosm (Brusseau et al. 1999). This pore water exposure route hypothesis effectively states that species specific degradation rates obtained from batch microcosms can be used to predict degradation rate in porous media with flowthrough. However, this hypothesis could be invalid if mass transport limitations between the mobile phase and the microorganisms reduce the efficiency of the reaction in a flowthrough matrix compared to batch systems (Simoni et al. 2001; Langner et al. 1998). In addition, biomass can be removed from the porous matrix by the flow, increasing the lagtime of the degradation reaction compared to batch systems (Sandrin et al. 2001).
There are only few studies which compare $\mathrm{CAH}$ degradation between batch and column systems to identify rate limiting physical or microbiological factors. Previous research demonstrated a significantly larger $0^{\text {th }}$ order TCE degradation rate in a porous matrix than in a homogeneous liquid culture but a correct numerical analysis was limited since the identity of the TCE degrading bacteria was unknown (Haest et al. 2008). Current advances in molecular microbiology enable the determination of species specific degradation rates, i.e. a quantification of the degradation per cell of one specific bacterial species, and its concurrent growth at the expense of the degraded substrate. As such, degradation activity can be distinguished from growth (He et al. 2003; Rahm et al. 2006; Smits et al. 2004). Schaefer et al. (2009) examined the reactive transport of cis-DCE in columns and performed batch tests with the initial column inoculum. The batch species specific degradation rates largely underestimated degradation in the column, i.e. the degradation rate per cell was larger in the column than in the batch experiment. A subsequent batch experiment with the effluent of the column showed a species specific degradation rate that was 200-times larger compared to that of the initial inoculum but corresponded to the observed dechlorination in the columns. This suggests that the cells gained activity in the porous matrix and this activity was not lost after elution of the cells out of the porous matrix. No additional data were available to explain the discrepancy in degradation activity between the initial inoculum and that of the column effluent. However, we note that the number of Dehalococcoides (cells $\mathrm{L}^{-1}$ ) in their initial batch experiment was $10^{3}-10^{4}$ larger than in the batch experiment with the column effluent. This effectively required a large extrapolation of the degradation rates per cell determined in the initial batch experiment to model the column data. The

Please cite this article as: P.J. Haest, J. Philips, D. Springael, E. Smolders (2011). The reactive transport of trichloroethene is influenced by residence time and microbial numbers. Journal of Contaminant Hydrology, 119: 89-98. 
species specific degradation rates potentially reduce with increasing cell numbers in this wide cell number interval due to competition effects not included in the modelling.

The objective of this work is to quantitatively assess the influence of the flow-through on biodegradation in the reactive transport of TCE. It is postulated that cells have an equal reactivity in a porous matrix as in liquid. As such, the dechlorination rate in a flowthrough porous matrix can be described by the species specific dechlorination rate observed in a liquid batch unless mass transport limitations prevail. To assess the research hypothesis, liquid batch tests and continuous flow-through column experiments were performed with varying flow rates and with stop-flow events. Quantitative real-time PCR (qPCR) was used to measure the dechlorinating species allowing to discern the species specific degradation rate from metabolic growth. This direct determination of the cellular activity allows for a better comparison between experimental setups than an indirect deduction of cellular numbers by plate counting techniques or an estimation of the yield coefficient from the measured metabolites in a pure culture. Other factors that could influence the dechlorination reaction such as the electron donor, $\mathrm{pH}$ and redox potential were experimentally controlled.

\section{Material and methods}

\subsection{The dechlorinating culture and medium preparation}

The KB-1 culture was kindly provided by SiREM (Ontario, Canada). This culture dechlorinates PCE and TCE to ethene. It contains Geobacter spp. and Dehalococcoides spp., among a wide variety of other microorganisms (Duhamel and Edwards 2006, 2007; Duhamel et al. 2002). Cultures used for inoculating the experiments were grown on 1 $\mathrm{mM}$ TCE and $1.5 \mathrm{mM}$ butyrate at $20{ }^{\circ} \mathrm{C}$ in an anaerobic mineral medium which is described in the supporting information. Butyrate was added as electron donor in a 5-fold electron equivalent (eeq) excess assuming fermentation to acetate and a degradation of $1 \mathrm{mM}$ TCE to ethene (Fennell \& Gossett 1998; Yang \& McCarty 1998).

\subsection{Batch experiment}

Liquid batch degradation experiments were initiated by inoculating $75 \mathrm{~mL}$ of the medium described previously in $160 \mathrm{~mL}$ vials under $\mathrm{N}_{2}$ atmosphere with $5 \mathrm{~mL}$ of the KB-1 culture grown on $1 \mathrm{mM}$ TCE (see previous discussion). This yielded $2.9 \times 10^{7}$ cells $\mathrm{L}^{-1}$ Geobacter and $2.9 \times 10^{8}$ cells $\mathrm{L}^{-1}$ Dehalococcoides at the start of the experiment.. TCE was added from a pure stock solution using a gastight glass syringe to a calculated aqueous concentration of $1 \mathrm{mM}$ taking into account the dimensionless Henry constants reported by Gossett (1987). Butyrate was added to $7.5 \mathrm{mM}$. The batch bottles were incubated in darkness on a horizontal shaker at $100 \mathrm{rpm}$ at $20^{\circ} \mathrm{C}$. The CAHs were measured in $1 \mathrm{~mL}$ liquid samples. The extracted volume was replenished using sterile $\mathrm{N}_{2}$.

\subsection{Flow-through experiment}

The column set-up consisted of 7 borosilicate glass columns with a length of $30 \mathrm{~cm}$ and an inner diameter of $5 \mathrm{~cm}$ (Fig 1). The columns were fitted with 5 equally spaced glass sampling ports from which water was sampled by connecting a vacuum sampling vial. Sampling ports were filled with glass wool. A quartz sand $\left(d_{50}=260 \mu \mathrm{m}\right.$, see supporting information Table SI.1) obtained from Sibelco N.V. (Belgium) was used as solid phase. The sterilized sand was homogeneously packed with medium containing the KB-1 culture (see batch experiment). The medium contained 6.5 vol\% of KB-1 inoculum. The final nominal microbial numbers were $1.9 \times 10^{5}$ cells $\mathrm{kg}^{-1}$ dry weight (dw) Geobacter spp. and $1.5 \times 10^{6}$ cells $\mathrm{kg}^{-1} \mathrm{dw}$

\footnotetext{
Please cite this article as: P.J. Haest, J. Philips, D. Springael, E. Smolders (2011). The reactive transport of trichloroethene is influenced by residence time and microbial numbers. Journal of Contaminant Hydrology, 119: 89-98.
} 
Dehalococcoides spp. Columns were incubated without flow-through for 3 days. After this period, a flow-through was ensured from the bottom to the top of the saturated porous matrix in the columns using a peristaltic pump. Tubing and connections were made of Viton and Kynar respectively to minimize sorption or diffusive losses of chlorinated ethenes. The influent medium was identical to the medium used in the batch experiment with a TCE and butyrate concentration of 1 , respectively $7.5 \mathrm{mM}$ and was stored in a Tedlar bag. The effluent was also gathered in a Tedlar bag. Six biotic columns were pairwise operated in 3 treatments with different pore water velocities covering the range in well permeable aquifers $\left(3-9-12 \mathrm{~cm} \mathrm{~d}^{-1}\right.$ ) (OVAM
2002). One abiotic column operated at the intermediate flow rate to check for abiotic removal of TCE. The retardation of TCE and cis-DCE was not examined in detail since the porous matrix consists of quartz sands with a negligible organic carbon content (Sibelco N.V., Belgium). Columns were operated in darkness at $20^{\circ} \mathrm{C} \pm 0.5^{\circ} \mathrm{C}$ during 247 days. The operational history is schematized in Table 1. At day 95, columns were flushed with 1 pore volume (PV) of sterile anaerobic demineralised water and re-inoculated via the influent because the degradation did not proceed largely (see results). The second inoculum was loaded for 1 PV yielding an extra $3.7 \times 10^{8}$ cells $\mathrm{kg}^{-1} \mathrm{dw}$ Geobacter and $1.1 \times 10^{9}$ cells kg ${ }^{-1} \mathrm{dw}$ Dehalococcoides in each column. Columns were equilibrated without

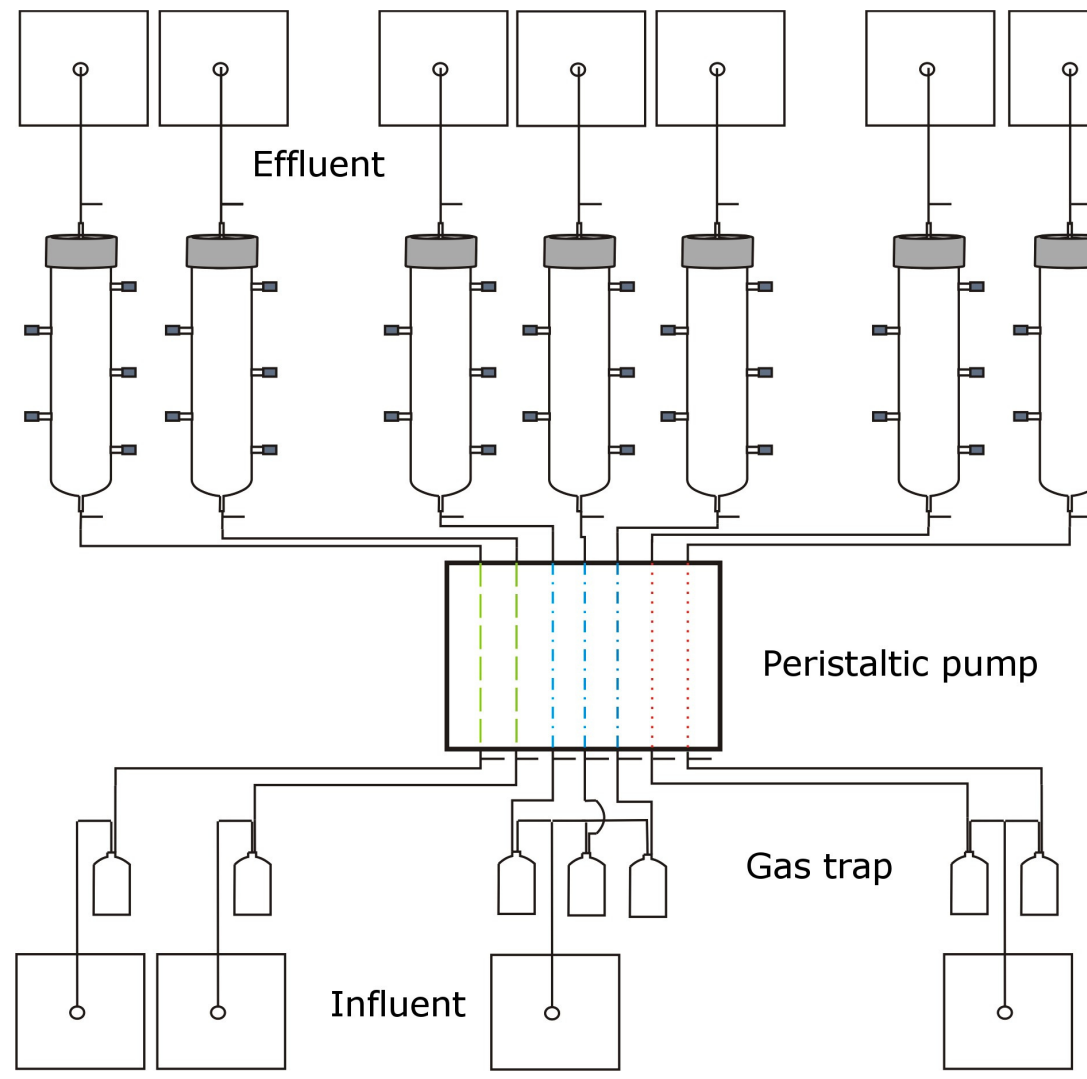

Figure 1: The column experiment consisted of 3 treatments with varying flow velocities of $12(--), 9(-),. 3(\ldots) \mathrm{cm} \mathrm{d}^{-1}$ and 2 replicates. One abiotic column was included to check for abiotic reactions and was operated at $9 \mathrm{~cm} \mathrm{~d}^{-1}$. Sterile anaerobic mineral medium was provided in 4 influent reservoirs. The medium passed a gas trap before it was pumped in the columns (upflow). The effluent of the columns was gathered in separate effluent reservoirs to measure the actual flow-through.

Please cite this article as: P.J. Haest, J. Philips, D. Springael, E. Smolders (2011). The reactive transport of trichloroethene is influenced by residence time and microbial numbers. Journal of Contaminant Hydrology, 119: 89-98. 
Table 1: The operational history of the column experiment with 3 treatments with varying pore water velocities covering the range in well-permeable aquifers. The columns were homogeneously inoculated and the experiment was initiated at day 0 with the respective pore water velocity. See text for more information.The $\mathrm{pH}$ in the high- and medium flow treatments decreased to 5 in days 187 to 191 due to an experimental error. The $\mathrm{pH}$ was restored to 6.8 at day 192 .

\begin{tabular}{llll}
\hline Day & \multicolumn{2}{l}{ Column Treatment } \\
& High-flow & Medium-flow & Low-flow \\
\hline $0-53$ & $12 \mathrm{~cm} \mathrm{~d}^{-1}$ & $9 \mathrm{~cm} \mathrm{~d}^{-1}$ & $3 \mathrm{~cm} \mathrm{~d}^{-1}$ \\
$54-55$ & Stop-flow & Stop-flow & Stop-flow \\
$56-63$ & $12 \mathrm{~cm} \mathrm{~d}^{-1}$ & $9 \mathrm{~cm} \mathrm{~d}^{-1}$ & $3 \mathrm{~cm} \mathrm{~d}^{-1}$ \\
$64-85$ & Stop-flow & Stop-flow & Stop-flow \\
$86-94$ & $12 \mathrm{~cm} \mathrm{~d}^{-1}$ & $9 \mathrm{~cm} \mathrm{~d}^{-1}$ & $3 \mathrm{~cm} \mathrm{~d}$ \\
95 & 2 nd inoculation & 2 nd inoculation & 2 nd inoculation \\
$96-101$ & Stop-flow & Stop-flow & Stop-flow \\
$102-109$ & $3 \mathrm{~cm} \mathrm{~d} d^{-1}$ & $3 \mathrm{~cm} \mathrm{~d}^{-1}$ & $3 \mathrm{~cm} \mathrm{~d}$ \\
$110-127$ & $12 \mathrm{~cm} \mathrm{~d}^{-1}$ & Stop-flow & $3 \mathrm{~cm} \mathrm{~d}^{-1}$ \\
$128-140$ & $12 \mathrm{~cm} \mathrm{~d}^{-1}$ & $9 \mathrm{~cm} \mathrm{~d}^{-1}$ & $3 \mathrm{~cm} \mathrm{~d}^{-1}$ \\
$141-147$ & Stop-flow & $9 \mathrm{~cm} \mathrm{~d}^{-1}$ & $3 \mathrm{~cm} \mathrm{~d}^{-1}$ \\
$148-203$ & $12 \mathrm{~cm} \mathrm{~d}^{-1}$ & $9 \mathrm{~cm} \mathrm{~d}^{-1}$ & $3 \mathrm{~cm} \mathrm{~d}^{-1}$ \\
203 & Stop-flow & Stop-flow & Stop-flow \\
$204-209$ & Stop-flow & Stop-flow & $3 \mathrm{~cm} \mathrm{~d}^{-1}$ \\
211 & $12 \mathrm{~cm} \mathrm{~d}^{-1}$ & $9 \mathrm{~cm} \mathrm{~d}^{-1}$ & Stop-flow \\
$212-247$ & $12 \mathrm{~cm} \mathrm{~d}^{-1}$ & $9 \mathrm{~cm} \mathrm{~d}^{-1}$ & $3 \mathrm{~cm} \mathrm{~d}^{-1}$ \\
\hline
\end{tabular}

flow-through from day 95 till day 101. Samples for CAH and qPCR analysis were obtained at regular time intervals during the experiment. Microbial numbers in the porous matrix at the end of the experiment were measured by analyzing wet sand samples taken at each sampling port and from the column in- and outlet.

A tracer test was carried out to examine the flow-through in the columns (see supporting information, Fig SI.1). Results of the tracer test together with experimental observations of $\mathrm{CAH}$ degradation during stop-flow events at the end of the column experiment show that there was no significant channelling in the columns.

\subsection{Analytical methods and data analysis} TCE, cis-dichloroethene (cis-DCE), vinylchloride (VC), ethene (ETH) and methane were measured in aqueous samples. The aqueous sample was analyzed by headspace analysis using a TriPlus autosampler and a Focus GC-FID (Thermo-Electron Corporation) equipped with an Rt-QPLOT column $(0.53 \mathrm{~mm}$ x $30 \mathrm{~m}$ ). Calibration curves were obtained from external standards. Detection limits of $0.006,0.011,0.057,0.005$ and $0.006 \mathrm{mM}$ were obtained for TCE, cis-DCE, VC, ETH and methane, respectively.

Butyrate, acetate, and bromide were measured by ion chromatography (Dionex ICS2000) equipped with an RFIC and an ECD detector. Ten microliters of diluted sample was injected on an AS11HC column $(2 \mathrm{~mm}$ i.d.) where separation was obtained using a $\mathrm{KOH}$ concentration gradient. Calibration curves were obtained from external standards with detection limits of $0.003,0.2$ and $1.5 \mathrm{mM}$ for bromide, butyrate respectively acetate.

The $\mathrm{pH}$ at the column effluent was regularly verified using $\mathrm{pH}$-indicator strips (Universal indicator, Merck).

DNA was extracted from $400 \mu \mathrm{L}$ of liquid sample or $400 \mu \mathrm{g}$ of wet solid phase as described by Uyttebroek et al. (2006). Quantification of Dehalococcoides numbers was performed using real-time PCR as described previously (Dijk et al. 2008) with a detection limit of $1.6 \times 10^{6} 16 \mathrm{~S}$ rDNA copies $\mathrm{L}^{-1}$. Following the same protocol, Geobacter and Bacteria numbers were quantified in duplicate using the ABsolute ${ }^{T M}$ QPCR SYBR Green Mix (ABgene) and primer sets Geo73f/Geo485r (Duhamel and Edwards, 2006) and Eub341F/Eub534r (Muyzer et al. 1993), respectively (see supporting information).

Microbial numbers were measured in the effluent (and pore water) of the column treatment at seven (five) times during the experiment. Three (one) measurements were

\footnotetext{
Please cite this article as: P.J. Haest, J. Philips, D. Springael, E. Smolders (2011). The reactive transport of trichloroethene is influenced by residence time and microbial numbers. Journal of Contaminant Hydrology, 119: 89-98.
} 
made before the re-inoculation and four (four) measurements were obtained after this second inoculation. Microbial growth of the different species in the column experiment was calculated based on the numbers measured in the column matrix at the end of the experiment plus the cumulative numbers measured in the effluent minus the inocula added. The yield of Geobacter numbers at the expense of TCE degradation in the column experiment was calculated from the cumulative $\mathrm{CAH}$ concentrations in the effluent.

Zero $^{\text {th }}$ order degradation rates were calculated for the dechlorination reaction. Zero $^{\text {th }}$ order kinetics were warranted as $\mathrm{CAH}$ concentrations were at least an order of magnitude higher than reported half velocity constants of 0.02 to $0.1 \mathrm{mM}$ (Harkness et al. 1999; Yu et al. 2005) and since first order kinetics were not observed in the batch experiment (see results). Dechlorination rates determined in the liquid batch experiment are calculated from the maximal degradation rates observed and dechlorination rates in the column experiment were calculated from measurements between successive sampling ports taking into account the mean travel time between the ports in the last week of the column experiment. The observed degradation rate is normalized to the amount of degrading cells that were measured yielding a species specific degradation rate, i.e. the degradation rate per cell of the degrading species. The flow-through species specific degradation rate in the columns was only calculated for the last week of the experiment since microbial numbers in the porous matrix were measured at the end of the experiment and these numbers were assumed to be representative for that period. The measured microbial numbers in the water saturated porous matrix were converted to pore volume basis to calculate the degradation rate per cell since TCE is present in the pore water and the comparison to batch degradation is made as mass degraded per volume of liquid. This conversion does not assume that no bacteria are attached to the sand but that attached cells have an equal reactivity as the pelagic cells, i.e. no mass transfer limitations are present. $A 0^{\text {th }}$ order degradation rate was calculated from the measured cis-DCE concentrations at the first sampling port in the low-flow column at the start and stop of the no-flow period.

\section{Results}

\subsection{Batch experiment}

TCE degradation in liquid culture was associated with growth of Geobacter and Dehalococcoides at the expense of TCE and cis-DCE degradation, respectively (Fig 2). Dechlorination of the daughter product cisDCE started when TCE was depleted. Degradation to ethene was not observed within the experimental time frame. However, degradation to ethene did occur in the source cultures illustrating that this potential was present in the inoculum (data not shown). Total bacterial numbers increased significantly compared to those of the specific dechlorinators at the start of the experiment which can be attributed to the proliferation of fermentation reactions. Surprisingly, no detectable depletion of butyrate was observed.The yeast extract in the medium $\left(0.05 \mathrm{~g} \mathrm{~L}^{-1}\right)$ could have provided a stoichiometric electron equivalent to fully degrade $1.5 \mathrm{mM}$ TCE to ethene (Aulenta et al. 2005). Other studies with butyrate as electron donor for the dechlorination of PCE or TCE used less yeast extract and indicate a slow but complete degradation of butyrate (Aulenta et al. 2005; Fennell et al. 1997). The yeast extract most probably contains some compounds that are more readily available electron donors for the dechlorination reaction than the fermentation of butyrate. The amino acids in the yeast extract can be deaminated and fermented yielding a direct

Please cite this article as: P.J. Haest, J. Philips, D. Springael, E. Smolders (2011). The reactive transport of trichloroethene is influenced by residence time and microbial numbers. Journal of Contaminant Hydrology, 119: 89-98. 


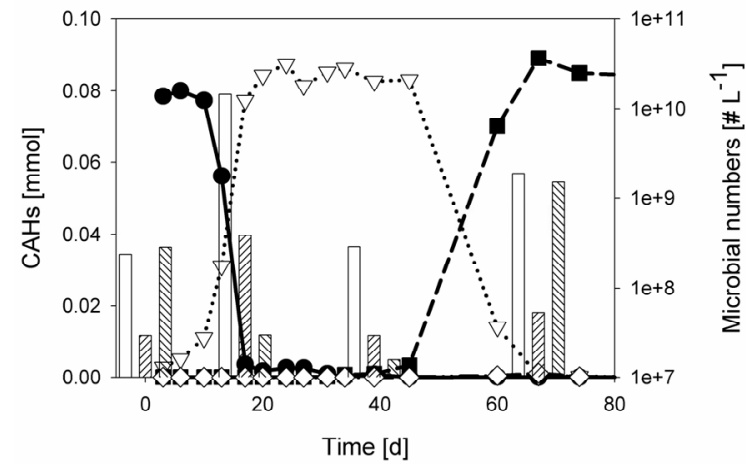

Figure 2: The CAH concentrations in the liquid batch experiment (-• TCE; .. $\nabla$ cis-DCE; --n VC; -..० ETH). The bacterial numbers are represented by the grouped vertical bars with Bacteria ( $\square$ ), Geobacter spp. ( ( ) and Dehalococcoides spp. (B), respectively.

energy source for dechlorinating organisms in case the organisms can perform this fermentation. Alternatively, the amino acids can be fermented by primary fermenters to volatile fatty acids which are converted by synthrophic bacteria to acetate and hydrogen (Mclnerney et al., 2009; Chen and Russell, 1988). This hydrogen can then be used by Dehalococcoides or other dechlorinating organisms that require hydrogen as the final electron donor in the reductive dechlorination.

The cis-DCE degradation was characterized by a long lag time pointing to the small degradation activity and the resulting slow growth of Dehalococcoides or, alternatively, to the necessity to modify growing conditions. Indeed, Dehalococcoides spp. pose strict nutrient requirements in the dechlorination reaction (He et al. 2007; Maymo-Gatell et al. 1997). In addition, cisDCE dechlorination by Dehalococcoides has been reported to require higher levels of $\mathrm{H}_{2}$ than those required for TCE degradation to cis-DCE (Lu et al. 2001).

\subsection{Column experiment}

No degradation of TCE was observed in the abiotic column (data not shown) and little or no TCE degradation was observed in the biotic columns during the initial 50 days of operation (Fig 3). The presence of cis-DCE in the effluent during the first PVs eluted indicates that dechlorination initially proceeded but was inhibited by the flowthrough. Effluent data indicated that the inoculum was largely flushed out in all treatments at the start of the experiment (see supporting information, Table SI.2). Two stop-flow events after day 50 (Table 1) initialized TCE degradation and TCE was degraded to a larger extent in the low-flow than in the high-flow treatment (Table 2). The differences in degradation rate between the flow treatments during the stop-flow are most likely related to the differences in microbial numbers in the columns: the total number of Geobacter and Dehalococcoides per column was calculated from pore water measurements and was significantly larger in the low-flow than in the high-flow column by day 87 , i.e. after the stop-flow events (Table 3). The tracer test indicated that the experimental set-up did not yield regions with stagnant water in the high- and medium-flow columns.

To stimulate dechlorination activity, columns were reinoculated at day 95. The dechlorination reaction proceeded to ethene in the low-flow treatment without a clear lagtime. In contrast, the dechlorination reaction only proceeded to cis-DCE in the mediumflow treatment and dechlorination did not proceed in the high-flow treatment where a new flow-interruption was required to initiate the reaction by day 150 . An accidental decrease of the influent $\mathrm{pH}$ to 5 on day 187 inhibited the dechlorination reaction in the high- and medium-flow treatments. When the $\mathrm{pH}$ was restored to 6.8 , dechlorination resumed after a flow-interruption with degradation of cis-DCE and VC occurring in the high-, respectively medium-flow treatment by the end of the column experiment.

Please cite this article as: P.J. Haest, J. Philips, D. Springael, E. Smolders (2011). The reactive transport of trichloroethene is influenced by residence time and microbial numbers. Journal of Contaminant Hydrology, 119: 89-98. 

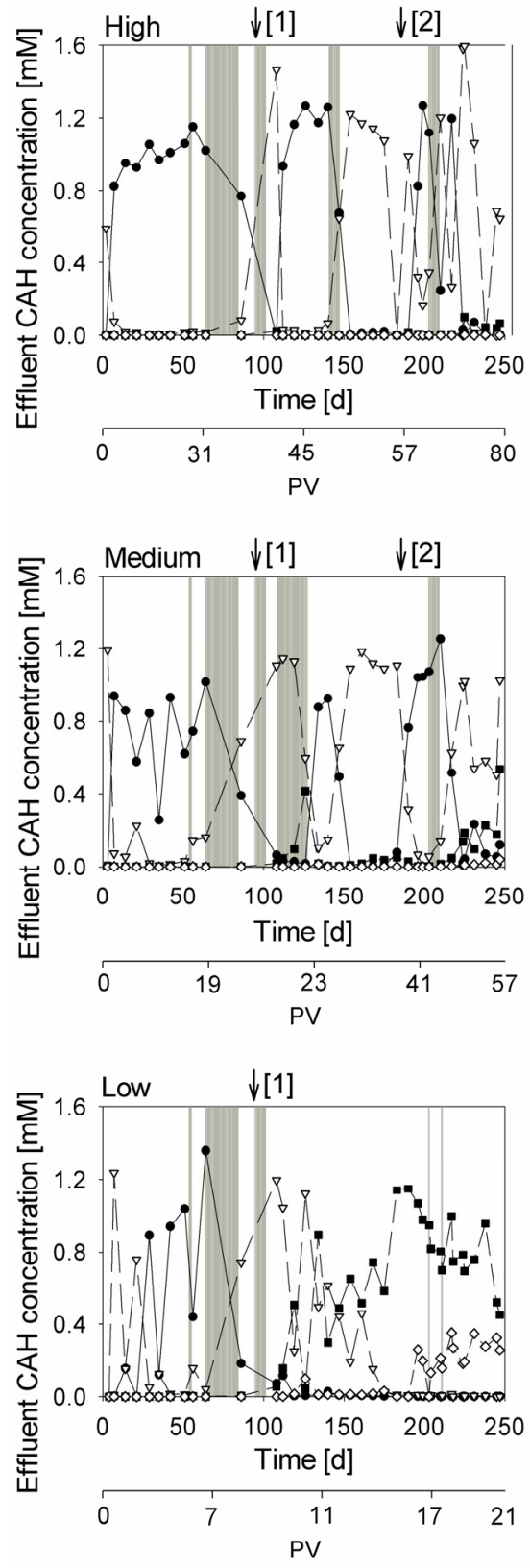

Figure 3: CAH concentrations in the effluent of the different flow treatments (- $\bullet$ TCE; .. $\nabla$ cis-DCE; -VC; -..O ETH). The second $x$-axis represents the number of pore volumes (PV) that passed through the column. Shaded vertical bars represent periods with no flow in the experiment. The second inoculation was performed at day 95 indicated by [1] and the $\mathrm{pH}$ decrease occurred at day 187 as indicated by [2]. The flow in the low-flow treatment was interrupted for 24 $h$ at day 203 and day 211.
Dechlorination proceeded in all column treatments during the last month, i.e. during the last 4-16 pore volumes. The extent of dechlorination in the columns during the last month of the experiment was significantly larger at lower flow rate (Table 2). No significant differences in gross dechlorination rates were found between flow rate treatments, i.e. the concentrations of chloride produced divided by the average residence times, suggesting that the dechlorination proceeded at the same rate in all flow treatments. However, such gross dechlorination rates combine the sequential steps in the dechlorination of TCE which are inherently different for the KB-1 culture. The maximal species specific TCE degradation rate was found to be 8 times larger than the respective cis-DCE degradation rate in previous research (Haest et al., 2010a; 2010b). Moreover, the dechlorination reaction was also heterogeneously distributed over the column length, i.e. mainly restricted to the column inlet in the high- and medium-flow column while it was well developed throughout the low-flow column (see further). The variability in dechlorination between the replicates of the high- and medium-flow columns is large but can be explained by the number of pore volumes that passed through: on average $20 \%$ PVs less passed through the $A$ replicates than through the $B$ replicates after the second inoculation due to experimental handling. Therefore, the dechlorination reaction proceeded to a larger extent in the $A$ replicates than in $B$ (see Table 2 ).

The stop flow events described previously promoted dechlorination and increased measured microbial numbers, which suggest an enhanced microbial growth under low or no flow. To exclude effects of microbial growth, the flow was interrupted for a short time of $24 \mathrm{~h}$ at day 203 and day 211 in the low-flow treatment where the dechlorination reaction was most stable. The cis-DCE

Please cite this article as: P.J. Haest, J. Philips, D. Springael, E. Smolders (2011). The reactive transport of trichloroethene is influenced by residence time and microbial numbers. Journal of Contaminant Hydrology, 119: 89-98. 
Table 2: Effluent composition at day 245 in each column replicate at a constant influent concentration of $1 \mathrm{mM}$ TCE. The extent of the dechlorination, i.e. chlorides formed in effluent, negatively relates to the pore water velocity, expressed as the number of PVs eluted after the $2^{\text {nd }}$ inoculation $\left(R^{2}=0.92\right)$.

\begin{tabular}{|c|c|c|c|c|c|c|}
\hline \multirow[t]{2}{*}{ Column treatment } & \multicolumn{4}{|c|}{$\begin{array}{c}\text { Effluent CAHs } \\
\text { [\% of the influent TCE concentration] }\end{array}$} & \multirow[t]{2}{*}{$\begin{array}{l}\text { Chlorides formed } * \\
{[\mathrm{mM}]}\end{array}$} & \multirow[t]{2}{*}{$\begin{array}{c}\text { Total } \mathrm{nr} \text { of PVs eluted after } \\
2^{\text {nd }} \text { inoculation }\end{array}$} \\
\hline & TCE & cis-DCE & VC & ETH & & \\
\hline High-flow A & 0.2 & 68.2 & 4.3 & 0.1 & 0.79 & 42.0 \\
\hline High-flow B & 25.0 & 79.5 & 0.2 & 0.1 & 0.80 & 52.9 \\
\hline Medium-flow A & 5.5 & 52.7 & 18.2 & 1.4 & 0.93 & 34.3 \\
\hline Medium- flow B & 3.8 & 74.9 & 1.1 & 0.0 & 0.77 & 41.4 \\
\hline Low-flow A & 0.0 & 0.1 & 61.9 & 38.8 & 2.40 & 3.90 \\
\hline Low-flow B & 0.1 & 0.1 & 74.2 & 46.7 & 2.89 & 2.50 \\
\hline
\end{tabular}

* The total dechlorination (chlorides formed) is calculated as cis-DCE+2*VC+3*ETH concentrations in the effluent.

Table 3: The microbial numbers in the column treatment at day 87 based on pore water measurements at the sampling ports along the column, and on day 247 based on measurements in the porous matrix. The yield of the TCE degrader Geobacter was calculated at day $247^{*}$.

\begin{tabular}{|c|c|c|c|c|c|}
\hline $\begin{array}{l}\text { Column } \\
\text { treatment }\end{array}$ & Day & $\begin{array}{c}\text { Bacteria } \\
\text { [cells column }{ }^{-1} \text { ] }\end{array}$ & $\begin{array}{c}\text { Geobacter } \\
\text { [cells column }{ }^{-1} \text { ] }\end{array}$ & $\begin{array}{l}\text { Dehalococcoides } \\
\text { [cells column }{ }^{-1} \text { ] }\end{array}$ & 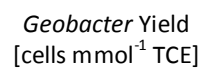 \\
\hline \multirow[t]{2}{*}{ High-flow } & 87 & $2.4 \times 10^{10}$ & $0.03 \times 10^{10}$ & $0.04 \times 10^{9}$ & \\
\hline & 247 & $3.4 \times 10^{10}$ & $0.09 \times 10^{10}$ & $1.7 \times 10^{9}$ & $0.9 \times 10^{9}$ \\
\hline \multirow[t]{2}{*}{ Medium-flow } & 87 & $1.7 \times 10^{10}$ & $0.4 \times 10^{10}$ & $0.02 \times 10^{9}$ & \\
\hline & 247 & $120 \times 10^{10}$ & $0.3 \times 10^{10}$ & $2.9 \times 10^{9}$ & $1 \times 10^{9}$ \\
\hline \multirow[t]{2}{*}{ Low-flow } & 87 & $33.1 \times 10^{10}$ & $1.6 \times 10^{10}$ & $4.1 \times 10^{9}$ & \\
\hline & 247 & $4.4 \times 10^{10}$ & $0.2 \times 10^{10}$ & $9.2 \times 10^{9}$ & $1 \times 10^{9}$ \\
\hline Flow rate effects ${ }^{* *}$ & & $* * *$ & $* * *$ & $* * *$ & n.s. \\
\hline
\end{tabular}

\footnotetext{
* The total yield of Geobacter on TCE degradation is determined from cumulative CAH concentrations and Geobacter numbers measured in the effluent, in the inoculum and in the column (whole matrix, i.e. wet porous matrix based) at the end of the experiment.

${ }^{* *}$ Determined by linear regression of the dependent variable in flow rate on day $87 ; * * *=p<0.001$; n.s. non significant at $p>0.5$.
}

degradation rate did not differ significantly between flow- and no-flow conditions $(0.15 \pm$ 0.11 vs. $0.16 \pm 0.11 \mathrm{mM} \mathrm{d}^{-1}$ ) suggesting that mass transport limitations between water and reactive sites, i.e. the degrading microorganisms, were small in this experiment.

Figure 4 shows the cumulative number of Geobacter and Dehalococcoides cells measured in the effluent by the end of the column experiment. The microbial numbers in the effluent were normalized to the net growth in the column since residence time largely affected the dechlorination and, hence, net growth. Ninety percent of the dechlorinators grown during $\mathrm{CAH}$ degradation were flushed out in the high-flow treatment while only 50 to $70 \%$ in the low-flow treatment. Flow-through negatively influenced the dechlorination reaction by depleting the column of the dechlorinators, as such enlarging the microbial lag time with increasing pore water velocities.

The CAH concentrations and microbial numbers in the porous matrix were measured

Please cite this article as: P.J. Haest, J. Philips, D. Springael, E. Smolders (2011). The reactive transport of trichloroethene is influenced by residence time and microbial numbers. Journal of Contaminant Hydrology, 119: 89-98. 


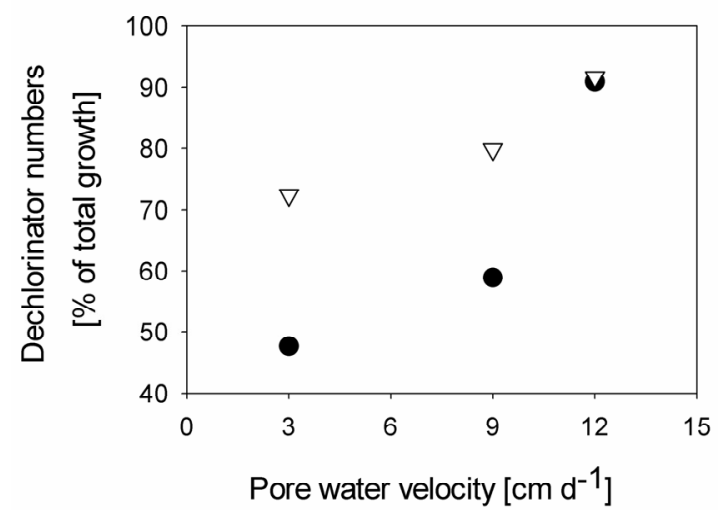

Figure 4: The cumulative number of dechlorinating species in the effluent of the column treatments (Geobacter $\bullet$ and Dehalococcoides $\nabla$ ).

throughout the columns at the end of the experiment (Fig 5 and Table 3). Microbial numbers correlated well with the dechlorination reaction: largest bacterial numbers were found at the column inlet where butyrate degradation occurred, high numbers of the TCE degrader Geobacter were found where TCE degradation was observed and high numbers of Dehalococcoides cells where cis-DCE and VC were degraded. A similar spatial distribution of Dehalococcoides was observed in a recent continuous-flow column study with the Evanite culture by Azizian et al. (2008). The Geobacter numbers measured in the wet, saturated porous matrix at the end of the experiment were on average 1.6 ( \pm standard deviation of 3.3) times higher than in the pore water sampled at corresponding adjacent locations. For Dehalococcoides this ratio amounted to 4.4 \pm 6.8 . No differences were observed between flow treatments. This ratio indicates that a significant fraction of cells were in solution. The large variability in these ratios is likely related to the spatial variability of cell numbers along the column (see Fig 5 ) and the different position of sampling: pore water was sampled at the outer edge of the column while matrix samples were taken from the centre. The apparent decline of the total
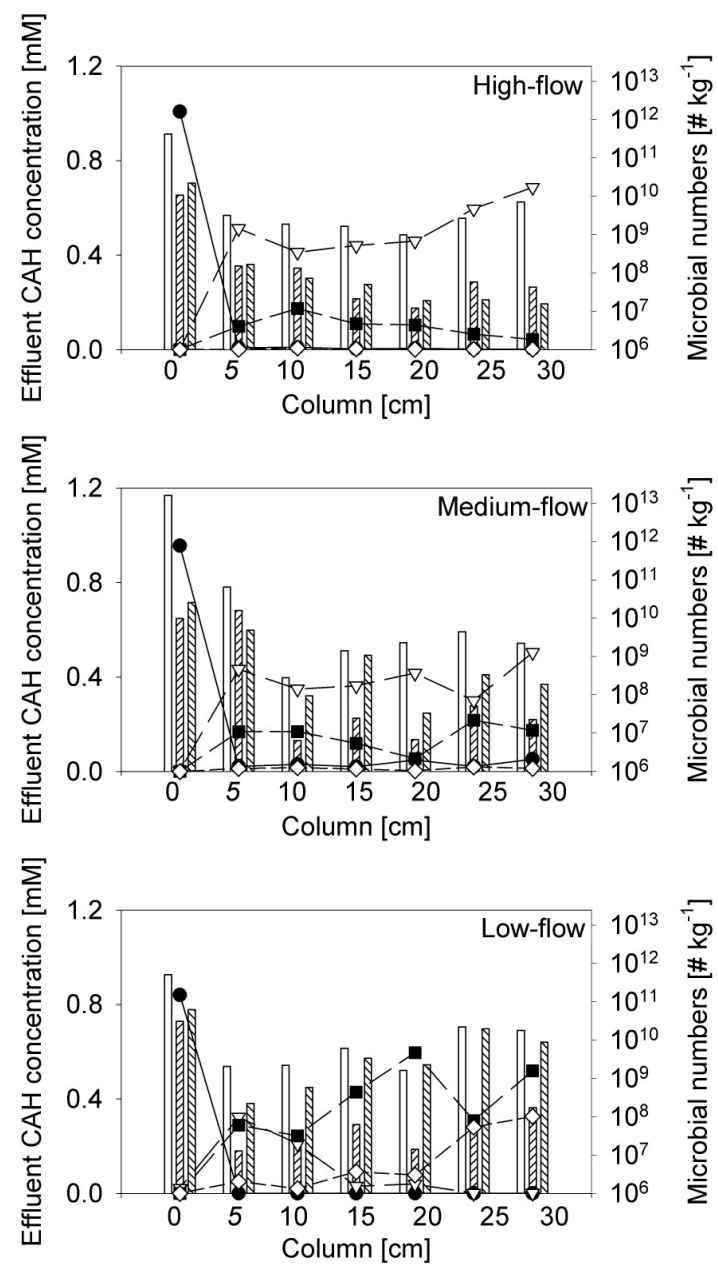

Figure 5: CAH concentrations throughout the 3 column treatments at the end of the column experiment for the high-flow, medium-flow and low-

flow treatment (-๑ TCE; .. $\nabla$ cis-DCE; --n VC; -.. ETH). The bacterial numbers are represented by the grouped vertical bars with Bacteria ( () ), Geobacter $\operatorname{spp} .(\square)$ and Dehalococcoides spp. $(\mathbb{Q})$, respectively.

bacterial number and the Geobacter number in the low-flow column at the end of the column experiment compared to day 87 (20 days no-flow) (see Table 3 ), is most likely due to the spatial distribution of degradation in the columns. TCE was not completely degraded in the low-flow column at day 87 indicating that TCE degradation could take place along the column with elevated

Please cite this article as: P.J. Haest, J. Philips, D. Springael, E. Smolders (2011). The reactive transport of trichloroethene is influenced by residence time and microbial numbers. Journal of Contaminant Hydrology, 119: 89-98. 
Geobacter numbers in the pore water. The TCE was already degraded by the first sampling port $(5 \mathrm{~cm})$ in all column treatments at the end of the experiment. The highly reactive zone near the column inlet was probably only a few $\mathrm{mm}$ long in the low-flow column. This very small scale was not represented by the matrix sample and the reported numbers for the low-flow column are probably an underestimation of the high bacterial numbers near the column inlet.

A partial fermentation of butyrate to acetate was observed in all column treatments after the second inoculation, together with the onset of the dechlorination reaction (data not shown). The extent of butyrate degradation did not differ between the column treatments. On average $1.5 \pm 0.5 \mathrm{mM}$ (of the $7.5 \mathrm{mM}$ in total) butyrate was degraded to acetate yielding enough electron equivalents for a complete degradation of TCE to ethene. No degradation of butyrate was observed beyond the first sampling port where cell numbers were lower than at the inlet. The bacterial numbers in the batch experiment were at least 10 times smaller than near the inlet of the columns which may explain the detectable butyrate fermentation in the columns in contrast with the batch setup. The supply of the electron donor is an important aspect of the subsequent degradation reactions and this study cannot exclude that the dechlorination reaction rate was influenced by the slow supply of electron donor beyond the first sampling port where no butyrate degradation was observed.

\section{Discussion: species specific degradation rates}

TCE was degraded from $1 \mathrm{mM}$ at the column inlet to below detection limit $(6 \mu \mathrm{M})$ at the first sampling port $(5 \mathrm{~cm})$ in all flow treatments. This suggests that TCE was degraded at shorter travel distances than 5 $\mathrm{cm}$ and thus degraded in a shorter time span than the travel time between the column inlet and the first sampling port. This means that the TCE degradation rate estimated from the column studies is an underestimated value (Table 4). Therefore, the TCE degradation rate of batch and column experiments was compared only for data

Table 4: The lumped $\left(k_{0, x}\right)$ and normalized $\left(k_{0, x, b i o}\right) 0^{\text {th }}$ order degradation rates ( \pm standard deviations) in the different experimental set-ups corresponding to the high-flow (HF) and low-flow (LF) column treatment. Degradation rates followed by identical characters ( $A$ and $B$ ) show no difference in the respective degradation rate between the experimental set-ups (Duncan test, $\mathrm{P}<0.05$ ).

\begin{tabular}{ccccc}
\hline Experiment & CAH degraded & $\begin{array}{c}\mathrm{k}_{0, \mathrm{x}} \\
{\left[\mathrm{mM} \mathrm{d}^{-1}\right]}\end{array}$ & $\begin{array}{c}\text { Microbial species* } \\
{\left[\log \left(\text { cells } \mathrm{L}^{-1}\right)\right]^{* *}}\end{array}$ & $\begin{array}{c}\mathrm{k}_{0, \mathrm{x}, \text { bio }} \\
{\left[\mathrm{mmol}_{\mathrm{ce}} \mathrm{d}^{-1}\right]}\end{array}$ \\
\hline Liquid batch & TCE & $0.3 \pm 0.2^{\mathrm{A}}$ & $8.8 \pm 0.6$ & $4.5 \times 10^{-10} \pm 3.1 \times 10^{-10 \mathrm{~A}}$ \\
Column (HF) & TCE & $1.7 \pm 0.2^{\mathrm{B}}$ & $9.5 \pm 1.3^{* * *}$ & $1.2 \times 10^{-10} \pm 0.1 \times 10^{-10 \mathrm{~A}}$ \\
\hline Liquid batch & cis-DCE & $0.04 \pm 0.01^{\mathrm{A}}$ & $9.8 \pm 0.7$ & $1.6 \times 10^{-11} \pm 2.0 \times 10^{-11 \mathrm{~A}}$ \\
Column (LF) & cis-DCE & $0.29 \pm 0.10^{\mathrm{B}}$ & $10.0 \pm 1.7^{* * *}$ & $0.4 \times 10^{-11} \pm 0.1 \times 10^{-11 \mathrm{~A}}$ \\
\hline
\end{tabular}

${ }^{*}$ Geobacter spp. for TCE and Dehalococcoides spp. for cis-DCE.

** Microbial numbers were measured in wet porous medium but results were converted to pore volume basis for comparison with the liquid culture (see text).

${ }^{* * *}$ Degradation rates measured in the last week of the column experiment were normalized to the measured amount of specific species at the end of the column experiment.

Please cite this article as: P.J. Haest, J. Philips, D. Springael, E. Smolders (2011). The reactive transport of trichloroethene is influenced by residence time and microbial numbers. Journal of Contaminant Hydrology, 119: 89-98. 
from the high-flow treatment (shortest travel time). The cis-DCE was not degraded beyond the first sampling port in the high- and medium-flow treatment. As such, the cis-DCE degradation rate was only examined in the low-flow treatment where cis-DCE was readily degraded.

The ANOVA analysis of the $0^{\text {th }}$ order TCE and cis-DCE degradation rates between the batch and column experiments revealed that degradation rates are significantly larger in columns than in batch, differences ranging between factors 4 and $7(P<0.05$, Table 4). Similar results were obtained previously (Haest et al. 2008). In contrast, the $0^{\text {th }}$ order rate constants normalized to associated cell numbers in the liquid medium or the porous matrix are not significantly different. A positive Spearman correlation $(P<0.05)$ was found between the $0^{\text {th }}$ order TCE degradation rate and the Geobacter numbers in the 2 experimental set-ups (data not shown). It could not be verified if the flow velocity affected the TCE degradation rate in the columns since TCE was fully degraded by the first sampling port in all treatments. The flowthrough clearly did not affect bacterial growth since similar yields of $1 \times 10^{9}$ cells $\mathrm{mmol}^{-1}$ were found for Geobacter in all flow treatments (Table 3). The cis-DCE degradation rate in the first $5 \mathrm{~cm}$ of the column where high microbial numbers were measured did not differ significantly between the flow treatments, which also suggests that mass transport limitations were minimal in this set up $(P<0.05$, data not shown). However, cisDCE degradation rates further in the column were lower than those in the first $5 \mathrm{~cm}$ and differed between flow treatments. The different cis-DCE degradation rates between flow-treatments and within each column were correlated with the number of Dehalococcoides present (Spearman correlation, $\mathrm{P}<0.05)$. Differences in cell numbers between flow treatments are explained by cell elution at higher flow velocities as described previously. The concomitant decrease of the cis-DCE degradation rate at higher flow explains the apparent stop of the dechlorination reaction at a higher flow velocity. These findings suggest that the differences between batch and column $\mathrm{CAH}$ degradation rates can largely be explained by the specific biomass while mass transport limitations in the flowthrough porous matrix were minimal in this set-up.

A limitation of the supply of electron donor could have played part in the observed degradation rate but was likely less important than the flow rate since stop-flow events, i.e. the potential for a higher donor supply, did not significantly enlarge the observed dechlorination rate in the low-flow column. The low Dehalococcoides cell numbers downstream in the columns of the mediumand high-flow treatments indicate that the growth was limited and correlate to the observed halt of cis-DCE degradation beyond port 1 in these treatments (see above). In addition, Dehalococcoides could have degraded part of the TCE near the column inlet yielding higher cell numbers for the subsequent cis-DCE degradation near the column inlet. It illustrates the difficulties for using species specific degradation constants to describe dechlorination by a mixed culture if the degradation pathways are not clear. This is especially true if metabolic bacterial growth needs to be considered. The limited spatial distribution of TCE degradation in the low-flow column complicated the quantification of the TCE degrading species (see previous discussion). This could prove more difficult in heterogeneous systems requiring careful consideration in the application of species specific degradation rates. Additional difficulties for using species specific degradation rates are the microbial quantification (e.g. the specificity of the primers in the qPCR reaction for the considered degrading species) or other

Please cite this article as: P.J. Haest, J. Philips, D. Springael, E. Smolders (2011). The reactive transport of trichloroethene is influenced by residence time and microbial numbers. Journal of Contaminant Hydrology, 119: 89-98. 
processes such as microbial competition, donor fermentation etc. that are not included in the normalization of the observed degradation rate. See for example the problems that were encountered by Schaefer et al. (2009) (see previous discussion).

The spatial and temporal CAH concentrations measured in this experiment were well predicted in a modelling study using batch degradation kinetics (Haest et al., 2010c), i.e. assuming an equal reactivity of the dechlorinators in column as in batch experiments. In addition, the apparent equal activity of pelagic and matrix cells in the porous matrix implies that practically no mass transport limitations were present at the pore scale and that the pore space could be considered as a homogeneous liquid, mimicking a batch system. This conclusion is only valid for the homogeneous sand with high permeability used in this experiment and is not necessarily true for more heterogeneous systems. Numerical studies showed that concentration gradients can develop at the pore-scale yielding mass transfer limitations to the attached cells with a substantial impact on the reaction rate (Dykaar and Kitanidis 1996; Meile and Tuncay 2006). Thullner et al. (2002) also observed that microbial growth can substantially affect water flow in a porous matrix, most likely by the production of extracellular polymeric substances. As such, it should be recognized that the degradation rate can be influenced by other factors than cellular activity in a more complex system. The assumption of equal cellular activity can be used at the larger scale to obtain a first estimation of plume spreading whether or not taking into account other rate-limiting processes, e.g. heterogeneity and hydrodynamic mixing (Cirpka, 2002; Bauer et al., 2009). The description of microbial degradation with species specific parameters can prove especially useful to evaluate theoretical concepts in the reactive transport model. The combination of species specific degradation parameters with stop-flow events allowed to discern cellular activity and mass transfer limitations in this experiment.

\section{Conclusion}

Microbial numbers influence the $\mathrm{CAH}$ degradation rate in columns since these numbers explain the lag time for degradation, the flow rate effects on cis-DCE degradation, the spatial variability of $\mathrm{CAH}$ degradation in the columns and the batch-column comparison. Flow rate effects on dechlorination are mainly explained by residence time, however dechlorination rates in the matrix are additionally explained by the residing microbial numbers. These numbers depend, in turn, on flow rate since a larger flow rate markedly increased microbial transport out of the column. An influence of the electron donor supply on the observed microbial growth could not be excluded in this research. Mass transport limitations in the porous matrix are small, if at all present in this set-up. However, this experiment was carried out with coarse inert quartz sand while in finer textured substrates, cell elution could be less important and mass transport limitation more important than in the system used here.

\section{Acknowledgment}

We thank the anonymous reviewers for the helpful comments. This research was carried out at the KU Leuven and was funded by a Ph.D grant of the Institute for the Promotion of Innovation through Science and Technology in Flanders (IWT-Vlaanderen).

\section{References}

Adamson, D.T., McDade, J.M., Hughes, J.B., 2003. Inoculation of Dnapl Source Zone to Initiate Reductive Dechlorination of Pce. Environ. Sci. Technol. 37 (11), 2525-2533.

Aulenta, F., Gossett, J.M., Papini, M.P., Rossetti, S., Majone, M., 2005.

Please cite this article as: P.J. Haest, J. Philips, D. Springael, E. Smolders (2011). The reactive transport of trichloroethene is influenced by residence time and microbial numbers. Journal of Contaminant Hydrology, 119: 89-98. 
Comparative study of methanol, butyrate, and hydrogen as electron donors for longterm dechlorination of tetrachloroethene in mixed anerobic cultures. Biotechnol. Bioeng. 91 (6), 743-753.

Azizian, M.F., Behrens, S., Sabalowsky, A., Dolan, M.E., Spormann, A.M., Semprini, L., 2008. Continuous-flow column study of reductive dehalogenation of PCE upon bioaugmentation with the Evanite enrichment culture. J. Contam. Hydrol. 100 (1-2), 11-21.

Bauer, R.D., Rolle, M., Bauer, S., Eberhardt, C., Grathwohl, P., Kolditz, O., Meckenstock, R.U., Griebler, C., 2009. Enhanced biodegradation by hydraulic heterogeneities in petroleum hydrocarbon plumes. J. Contam. Hydrol. 105 (1-2), 56-68.

Bradley, P.M., 2003. History and ecology of chloroethene biodegradation: a review. Bioremediation Journal 7 (2), 81-109.

Brusseau, M.L., Hu, M.Q., Wang, J.M., Maier, R.M., 1999. Biodegradation during contaminant transport in porous media. 2. The influence of physicochemical factors. Environ. Sci. Technol. 33 (1), 96103.

Carr, C.S., Hughes, J.B., 1998. Enrichment of High Rate Pce Dechlorination and Comparative Study of Lactate, Methanol, and Hydrogen as Electron Dopers to Sustain Activity. Environ. Sci. Technol. 32 (12), 1817-1824.

Cirpka, O.A., 2002. Choice of dispersion coefficients in reactive transport calculations on smoothed fields. J. Contam. Hydrol. 58 (3-4), 261- 282.

Chen, G., Russell, J.B., 1988. Fermentation of Peptides and Amino Acids by a MonensinSensitive Ruminal Peptostreptococcus. Appl. Environ. Microb. 54(11), 2742-2749.

Christ, J.A., Ramsburg, C.A., Abriola, L.M., Pennell, K.D., Loffler, F.E., 2005. Coupling Aggressive Mass Removal With Microbial Reductive Dechlorination for Remediation of Dnapl Source Zones: a Review and Assessment. Environ. Health Persp. 113 (4), 465-477.

Dijk, J.A., Breugelmans, P., Philips, J., Haest, P.J., Smolders, E., Springael, D., 2008. Catalyzed Reporter DepositionFluorescent in Situ Hybridization (CardFish) Detection of Dehalococcoides. J. Microbiol. Meth. 73 (2), 142-147.

Duhamel, M., Edwards, E.A., 2006. Microbial composition of chlorinated ethenedegrading cultures dominated by Dehalococcoides. FEMS Microbiol. Ecol. 58 (3), 538-549.

Duhamel, M., Edwards, E.A., 2007. Growth and yields of dechlorinators, acetogens, and methanogens during reductive dechlorination of chlorinated ethenes and dihaloelimination of 1,2-dichloroethane. Environ. Sci. Technol. 41 (7), 2303-2310.

Duhamel, M., Wehr, S.D., Yu, L., Rizvi, H., Seepersad, D., Dworatzek, S., Cox, E.E., Edwards, E.A., 2002. Comparison of Anaerobic Dechlorinating Enrichment Cultures Maintained on Tetrachloroethene, Trichloroethene, CisDichloroethene and Vinyl Chloride. Water Res. 36 (17), 4193-4202.

Dykaar, B.B., Kitanidis, P.K., 1996. Macrotransport of a Biologically Reacting Solute Through Porous Media. Water Resources Res. 32 (2), 307-320.

Ellis, D.E., Lutz, E.J., Odom, J.M., Buchanan, R.J., Bartlett, C.L., Lee, M.D., Harkness, M.R., Deweerd, K.A., 2000. Bioaugmentation for Accelerated in Situ Anaerobic Bioremediation. Environ. Sci. Technol. 34 (11), 2254-2260.

Fennell, D.E., Gossett, J.M., 1998. Modeling the Production of and Competition for Hydrogen in a Dechlorinating Culture. Environ. Sci. Technol. 32 (16), 2450-2460.

Fennell, D.E., Gossett, J.M., Zinder, S.H., 1997. Comparison of butyric acid, ethanol, lactic acid and propionic acid as hydrogen donors for the reductive dechlorination of

Please cite this article as: P.J. Haest, J. Philips, D. Springael, E. Smolders (2011). The reactive transport of trichloroethene is influenced by residence time and microbial numbers. Journal of Contaminant Hydrology, 119: 89-98. 
tetrachloroethene. Environ. Sci. Technol. 31 (3), 918-926.

Gossett, J.M., 1987. Measurement of Henrys Law Constants for C1 and C2 Chlorinated Hydrocarbons. Environ. Sci. Technol. 21 (2), 202-208.

Haest, P.J., Springael, D., Smolders, E., 2010a. Dechlorination kinetics of TCE at toxic TCE concentrations: Assessment of different models. Water Res. 44 (1), 331-339.

Haest, P.J., Springael, D., Smolders, E., 2010b. Erratum to "Dechlorination kinetics of TCE at toxic TCE concentrations: Assessment of different models" [Water Research 44 (2010) 1]. Water Res. 44 (9), 2995.

Haest, P.J., Springael, D., Smolders, E., 2010c. Modelling reactive $\mathrm{CAH}$ transport using batch experiment degradation kinetics. Water Res. 44 (9), 2981-2989.

Haest, P.J., Philips, J., Springael, D., Smolders, E., 2008. Challenges for Modeling Groundwater Remediation: Dehalogenation Kinetics of Trichloroethene Vary between Batch and Column Experiments. In: Sass B. M. (ed), Proceedings of the Sixth International Conference on Remediation of Chlorinated and Recalcitrant Compounds. Battelle, Columbus, $\mathrm{OH}$, www.battelle.org/chlorcon., Monterey, CA.

Harkness, M.R., Bracco, A.A., Brennan, M.J., Deweerd, K.A., Spivack, J.L., 1999. Use of Bioaugmentation to Stimulate Complete Reductive Dechlorination of Trichloroethene in Dover Soil Columns. Environ. Sci. Technol. 33 (7), 1100-1109.

He, J., Holmes, V.F., Lee, P.K., Alvarez-Cohen, L., 2007. Influence of vitamin B12 and cocultures on the growth of Dehalococcoides isolates in defined medium. Appl. Environ. Microb. 73 (9), 2847-2853.

He, J., Ritalahti, K.M., Aiello, M.R., Loffler, F.E., 2003. Complete Detoxification of
Vinyl Chloride by an Anaerobic Enrichment Culture and Identification of the Reductively Dechlorinating Population as a Dehalococcoides Species. Appl. Environ. Microb. 69 (2), 996-1003.

Langner, H.W., Inskeep, W.P., Gaber, H.M., Jones, W.L., Das, B.S., Wraith, J.M., 1998. Pore water velocity and residence time effects on the degradation of 2,4-D during transport. Environ. Sci. Technol. 32 (9), 1308-1315.

Lu, X.X., Tao, S., Bosma, T., Gerritse, J., 2001. Characteristic hydrogen concentrations for various redox processes in batch study. J. Environ. Sci. Heal. A 36 (9), 17251734.

Major, D.W., McMaster, M.L., Cox, E.E., Edwards, E.A., Dworatzek, S.M., Hendrickson, E.R., Starr, M.G., Payne, J.A., Buonamici, L.W., $2002 . \quad$ Field Demonstration of Successful Bioaugmentation to Achieve Dechlorination of Tetrachloroethene to Ethene. Environ. Sci. Technol. 36 (23), 5106-5116.

Maymo-Gatell, X., Chien, Y., Gossett, J.M., Zinder, S.H., 1997. Isolation of a bacterium that reductively dechlorinates tetrachloroethene to ethene. Science 276 (5318), 1568-1571.

Mclnerney, M.J., Sieber, J.R. Robert, P.G., 2009. Syntrophy in anaerobic global carbon cycles. Curr. Opin. Biotech. 20(6), 623-632.

Meile, C., Tuncay, K., 2006. Scale dependence of reaction rates in porous media. Adv. Water Resour. 29 (1), 62-71.

Muyzer, G., Dewaal, E.C., Uitterlinden, A.G., 1993. Profiling of Complex MicrobialPopulations by Denaturing Gradient GelElectrophoresis Analysis of Polymerase Chain Reaction-Amplified Genes-Coding for 16 s Ribosomal-Rna. Appl. Environ. Microb. 59 (3), 695-700.

Please cite this article as: P.J. Haest, J. Philips, D. Springael, E. Smolders (2011). The reactive transport of trichloroethene is influenced by residence time and microbial numbers. Journal of Contaminant Hydrology, 119: 89-98. 
OVAM. 2002. In Dutch: Code van goede praktijk - Pump \& Treat Deel 1: Grondwateronttrekkingsaspecten. OVAM.

Rahm, B.G., Chauhan, S., Holmes, V.F., Macbeth, T.W., Sorenson, K.S.J., AlvarezCohen, L., 2006. Molecular characterization of microbial populations at two sites with differing reductive dechlorination abilities. Biodegradation 17 (6), 523-534.

Sandrin, S.K., Jordan, F.L., Maier, R.A., Brusseau, M.L., 2001. Biodegradation during contaminant transport in porous media: 4. Impact of microbial lag and bacterial cell growth. J. Contam. Hydrol. 50 (3-4), 225-242.

Schaefer, C.E., Condee, C.W., S. V., Steffan, R.J., 2009. Bioaugmentation for chlorinated ethenes using Dehalococcoides sp.: Comparison ... Chemosphere 75 (2), 141-148.

Sleep, B.E., Seepersad, D.J., Mo, K., Heidorn, C.M., Hrapovic, L., Morrill, P.L., McMaster, M.L., Hood, E.D., LeBron, C., Lollar, B.S., Major, D.W., Edwards E.A., 2006. Biological enhancement of tetrachloroethene dissolution and associated microbial community changes. Environ. Sci. Technol. 40 (11), 3623-3633.

Simoni, S.F., Schäfer, A., Harms, H., Zehnder, A.J.B,. 2001. Factors affecting mass transfer limited biodegradation in saturated porous media. J. Contam. Hydrol. 50 (1-2), 99-120.

Smits, T.H.M., Devenoges, C., Szynalski, K., Maillard, J., Holliger, C., 2004. Development of a Real-Time Pcr Method for Quantification of the Three Genera Dehalobacter, Dehalococcoides, and Desulfitobacterium in Microbial Communities. J. Microbiol. Meth. 57 (3), 369-378.

Thullner, M., Mauclaire, L., Schroth, M.H., Kinzelbach, W., Zeyer, J., 2002. Interaction between water flow and spatial distribution of microbial growth in a twodimensional flow field in saturated porous media. J. Contam. Hydrol. 58 (1-2) 169- 189.

Uyttebroek, M., Breugelmans, P., Janssen, M., Wattiau, P., Joffe, B., Karlson, U., OrtegaCalvo, J.J., Bastiaens, L., Ryngaert, A., Hausner, M., Springael, D., 2006. Distribution of the Mycobacterium community and polycyclic aromatic hydrocarbons (PAHs) among different size fractions of a long-term $\mathrm{PAH}$ contaminated soil. Environ. Microbiol. 8 (5), 836-847.

Yang, Y.R., McCarty, P.L., 1998. Competition for hydrogen within a chlorinated solvent dehalogenating anaerobic mixed culture. Environ. Sci. Technol. 32 (22), 3591-3597.

Yang, Y.R., McCarty, P.L., 2002. Comparison Between Donor Substrates for Biologically Enhanced Tetrachloroethene Dnapl Dissolution. Environ. Sci. Technol. 36 (15), 3400-3404.

Yu, S., Dolan, M.E., Semprini, L., 2005. Kinetics and Inhibition of Reductive Dechlorination of Chlorinated Ethylenes by Two Different Mixed Cultures. Environ. Sci. Technol. 39 (1), 195-205.

Please cite this article as: P.J. Haest, J. Philips, D. Springael, E. Smolders (2011). The reactive transport of trichloroethene is influenced by residence time and microbial numbers. Journal of Contaminant Hydrology, 119: 89-98. 